\title{
Experimental study of the falling film of liquid around a Taylor bubble
}

\author{
Marcos B. de Azevedo ${ }^{\mathrm{a}, \mathrm{c}}$, Douglas dos Santos ${ }^{\mathrm{a}, \mathrm{b}}$, José L.H. Faccini ${ }^{\mathrm{a}}$, Jian Su ${ }^{\mathrm{c}, *}$ \\ ${ }^{a}$ Nuclear Engineering Division, Instituto de Engenharia Nuclear/CNEN, Rio de Janeiro, 21941-906, Brazil \\ ${ }^{\mathrm{b}}$ Nuclear Engineering Department, POLI, Universidade Federal do Rio de Janeiro, Rio de Janeiro, 21945-970, Brazil \\ c Nuclear Engineering Program, COPPE, Universidade Federal do Rio de Janeiro, CP 68509, Rio de Janeiro, 21941-972, Brazil
}

\section{A R T I C L E I N F}

\section{Article history:}

Available online 6 October 2016

\section{Keywords:}

Taylor bubble

Falling liquid film

Equilibrium film thickness

Film development length

Ultrasonic technique

Stagnant liquid

\begin{abstract}
A B S T R A C T
The present work reports an experimental study of the falling liquid film around single Taylor bubbles rising in vertical tubes filled with stagnant liquids by using a pulse-echo ultrasonic technique. The experiments were carried out in acrylic tubes $2.0 \mathrm{~m}$ long, with inner diameters of $0.019,0.024$ and $0.034 \mathrm{~m}$, with five water-glycerin mixtures, corresponding to inverse viscosity number ranging from 15 to 22422 . The rising bubble and the falling liquid film were measured by using ultrasonic transducers located at the one side of the tube. The velocity and profile of the Taylor bubble, and the development length and equilibrium thickness of the falling liquid film around the bubble were obtained by the ultrasonic signals processing. Based on the experimental results of the present study, several correlations available to estimate the equilibrium thicknesses of liquid films falling around Taylor bubbles were evaluated and new correlations were proposed to estimate the dimensionless equilibrium film thickness and the film development length respectively.
\end{abstract}

(c) 2016 Elsevier Ltd. All rights reserved.

\section{Introduction}

Gas-liquid two-phase flow is an important and complex form of multiphase flows and is frequently encountered in a whole range of industrial applications such as nuclear reactor cooling systems, pipeline systems for the transport of oil-gas mixtures, evaporators, boilers, condensers, among others. Slug flow is one of the common flow patterns in gas-liquid two-phase flow and is characterized by long bullet-shaped bubbles, also called Taylor bubbles or elongated bubbles that occupy nearly the entire cross-section of the pipe and liquid slugs between successive bubbles. The liquid moves around the bubbles in a thin film and expands at the rear of the bubble, inducing a liquid wake.

Flow parameters are very important for understanding the flow structure, which has stimulated the development of new methods and techniques able to measure them with a high degree of spatial and temporal resolutions. One of these techniques is the ultrasonic technique, which is non-intrusive, low costs and applicable at high pressure and high temperature conditions. Additionally, it can be applied to pipes and containers of different materials and to either optically transparent or opaque fluids. In the pulse-echo mode, the technique has been used to observe the location and size of bubbles, due to its ability to determine

\footnotetext{
* Corresponding author.

E-mail address: sujian@nuclear.ufrj.br (J. Su).
}

the gas-liquid interfaces (Murai et al., 2010; Park et al., 2015; Rahim et al., 2007). This method can also be applied in a liquid film thickness measurement and characterization of flow patterns (Chang and Morala, 1990; Matikainen et al., 1985). The knowledge of the Taylor bubble structure and its motion, including in stagnant liquid, is of fundamental importance in gas-liquid two-phase flow theory. Fig. 1 presents a schematic of a Taylor bubble rising in a stagnant liquid inside a vertical tube with inner radius $R$.

The pioneering works of Dumitrescu (1943) and Davies and Taylor (1950) studied, theoretically and experimentally, the propagation of infinite elongated bubbles in vertical tubes. The models proposed by them for the bubble shape did not predicted the achievement of an equilibrium condition at the liquid film (developed film), when the liquid film thickness and velocity profile can be considered constant. Nicklin et al. (1962) reported a constant film thickness below a distance of six tube inner diameters from the tip of the bubble nose. Mao and Dukler (1991) used intrusive techniques and concluded that a constant thickness would never be reached in the liquid film.

More recently, Nogueira et al. (2006) used optical techniques to measure velocity profile characteristics of developed falling film and constant film thickness below a certain distance from the bubble nose. Llewellin et al. (2012) developed a dimensionless approach to study falling films and found that the dimensionless film thickness $\delta^{\prime}$ (the ratio of the film thickness $\delta$ to the tube radius $R$ ) is a function only of the dimensionless inverse viscosity number $N_{f}$. Single Taylor bubbles were generated by partially 


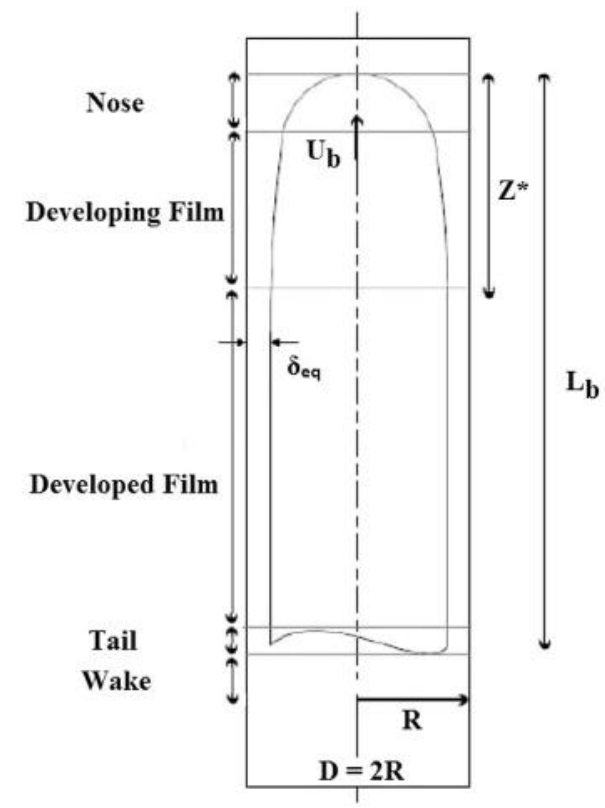

Fig. 1. Schematic of a Taylor bubble rising in a stagnant liquid inside a vertical tube.

filling the tubes with liquid to leave an air pocket of length $L_{0}$, then sealing and inverting the tubes. A relationship between the bubble length $L_{b}$ and the initial length of the air pocket $L_{0}$ was used to determine the film thickness. A systematic validation of several correlations for the thickness of falling films around Taylor bubbles was carried out in a wide range of $N_{f}$.

The knowledge about the liquid film development is very relevant when the slug flow hydrodynamic model is used for a better understanding of the processes of heat and mass transfer (Ghajar, 2005) and of the mechanisms of interaction between two consecutive Taylor bubbles (Campos and Guedes de Carvalho, 1988). Therefore, it is important to further the study of the equilibrium condition of liquid films around Taylor bubbles, adding data of measurements of the equilibrium film thicknesses and the distances from the bubble noses where the films reach the equilibrium by using different techniques.

In the present work, the pulse-echo ultrasonic technique was used to measure the rising bubble velocity $U_{b}$, the film development length $Z^{*}$, and the equilibrium thickness $\delta_{e q}$ of the falling liquid film around Taylor bubbles rising in stagnant liquid vertical columns sealed at the ends and with different inner diameters. Water, glycerin and water-glycerin solutions were used as working fluids. The experimental results obtained were used to evaluate several correlations available in the literature, applying a theoretical approach proposed by Llewellin et al. (2012). Based on the analysis and experimental results, new empirical correlations were proposed to estimate the equilibrium thickness $\delta_{e q}$ and the film development length $Z^{*}$ respectively.

\section{A brief literature review}

When the liquid film around a Taylor bubble is thin, the local curvature of the tube can be neglected and the behavior of the liquid film can be considered as the same of a liquid falling down a planar surface (Brown, 1965; Goldsmith and Mason, 1962). The thin film assumption is applicable in situations involving rising Taylor bubbles in tubes filled with water or other low viscosity liquids.

Liquid films are usually described in the literature by using the film Reynolds number $R e_{f}$, defined as (Karapantsios and Karabelas,
1995; Llewellin et al., 2012):

$\operatorname{Re}_{f}=4 \frac{\rho_{L} v_{f} \delta}{\mu_{L}}$.

where $\mu_{L}$ is the liquid viscosity, $\rho_{L}$ the liquid density, $\delta$ the film thickness, and $v_{f}$ the film velocity. On the other hand, the bubble Reynolds number $R e_{b}$ can be used to characterize the Taylor bubbles:

$\operatorname{Re}_{b}=\frac{\rho_{L} U_{b} D}{\mu_{L}}$.

where $U_{b}$ is the rising bubble velocity and $D$ is the tube inner diameter.

Furthermore, it is possible to write $R e_{b}$ as a function of the dimensionless inverse viscosity number $N_{f}$ and the bubble Froude number:

$\operatorname{Re}_{b}=N_{f} F r_{b}$

where $F r_{b}=U_{b} / \sqrt{g D}$ and $g$ is the gravitational acceleration.

The dimensionless inverse viscosity number $N_{f}$ can be obtained by combining the Eotvos number $\left(E 0=\rho_{L} g D^{2} / \sigma_{L}\right)$ and the Morton number $\left(M=g \mu_{L}^{4} /\left(\rho_{L} \sigma_{L}^{3}\right)\right)$ to eliminate the surface tension $\sigma_{L}$ (Llewellin et al., 2012; Wallis, 1969):

$N_{f}=\left[\frac{E o^{3}}{M}\right]^{1 / 4}=\frac{\rho_{L}}{\mu_{L}} \sqrt{g D^{3}}$.

The parameter $N_{f}$ is useful to characterize the Taylor bubbles, using only the fluid properties and the tube diameter, and is also called the buoyancy Reynolds number by some authors (Viana et al., 2003).

Under the thin film assumption $(\delta<<R)$, Llewellin et al. (2012) showed that $R e_{b}=R e_{f}$ for Taylor bubbles rising inside vertical cylindrical tubes. Correlations for the thickness of film falling down a surface written originally in function of $R e_{f}$ can be rewritten in terms of the dimensionless inverse viscosity number $N_{f}$, the bubble Reynolds number $R_{b}$, and the Froude number $F r_{b}$ to estimate the dimensionless thickness, $\delta^{\prime}=\delta / R$ :

- Nusselt (1916) Correlation:

$$
\delta^{\prime}=\left(6 \frac{R e_{b}}{N_{f}^{2}}\right)^{1 / 3},
$$

- Karapantsios and Karabelas (1995) Correlation:

$$
\delta^{\prime}=\frac{0.428 R_{b}^{0.538}}{\sqrt[3]{N_{f}^{2}}}
$$

- Lel et al. (2005) Correlation:

$$
\delta^{\prime}=\frac{2+0.641 R e_{b}^{0.47}}{\sqrt[3]{N_{f}^{2}}} .
$$

Based on the observation that $F r_{b}$ is largely independent of Eo for $E o>40$, Llewellin et al. (2012) proposed a simplification for the general empirical correlation presented by Viana et al. (2003), when the surface tension $\sigma_{L}$ is negligible:

$F r_{b}=0.34\left[1+\left(\frac{31.08}{N_{f}}\right)^{1.45}\right]^{-0.71}$.

Whereas these approaches are valid, Eqs. (3) and (8) indicate that $\mathrm{Fr}_{b}$ and $R e_{b}$ are only functions of $N_{f}$ when Eo $>40$, allowing that $\delta^{\prime}$ can be written as a function of $N_{f}$ only, in Eqs. (5)-(7).

Goldsmith and Mason (1962) applied the Navier-Stokes equation for laminar flow, assuming a thin film, and obtained a 
relationship between the rising bubble velocity $U_{b}$ and the liquid film thickness $\delta$ around a Taylor bubble:

$U_{b}=\frac{2 \rho_{L} g \delta^{3}}{3 \mu_{L} R}$.

These authors observed that Eq. (9) was already derived by Nusselt (1916) and, in fact, if this correlation is rearranged, it recovers the dimensionless form, defined by Eq. (5).

Extending the analysis of Goldsmith and Mason (1962), without the thin film assumption, Brown (1965) also related $U_{b}$ and $\delta$ and proposed a correlation to estimate $\delta$ that, in dimensionless form, can be written as (Llewellin et al., 2012):

$\delta^{\prime}=2 \frac{-1+\sqrt{1+2.44 N_{f}^{2 / 3}}}{2.44 N_{f}^{2 / 3}}$.

Another correlation to estimate the thicknesses of films around elongated bubbles in stagnant liquids is due to Kang et al. (2010), obtained from numerical simulations:

$\delta^{\prime}=0.64 N_{f}^{-0.2}$.

Llewellin et al. (2012) determined the equilibrium liquid film thicknesses around Taylor bubbles and proposed an empirical correlation, based on the combined results of their measurements and those from Nogueira et al. (2006):

$\delta^{\prime}=0.204+0.123 \tanh \left(2.66-1.15 \log N_{f}\right)$.

Another important parameter related with liquid films falling around Taylor bubbles is the film development length $Z^{*}$, that defines the minimum bubble length required to allow that the film reaches its equilibrium condition. Sena Esteves and Guedes de Carvalho (1993) proposed a correlation to estimate $Z^{*}$ :

$\frac{Z^{*}}{D}=0.0852\left(F r_{b}^{2} N_{f}\right)^{2 / 3}$.

Thus, considering correct the previously mentioned approaches of Viana et al. (2003) and Llewellin et al. (2012), Eq. (8) allows Eq. (13) to be rewritten with $Z^{*} / D$ as a function of $N_{f}$ only.

\section{Experimental apparatus and procedures}

The experimental data were obtained from vertical columns partially filled with stagnant liquid. The vertical columns consisted of acrylic tubes with $2.0 \mathrm{~m}$ in length and inner diameters of $0.019,0.024$ and $0.034 \mathrm{~m}$ sealed at the ends. A Taylor bubble with length $L_{b}$ was formed by the inversion $\left(t_{1}-t_{2}\right)$ of the pipe partially filled with liquid to leave an air pocket of length $L_{0}$, as illustrated in Fig. 2b.

The acrylic tubes were fixed and aligned on a metallic support which was rotated around a pivot. The tube was held in the vertical position by a small plate that limited the support rotation and allowed their positioning at the vertical position, as presented in Fig. 2a. The vertical position was determined by using a digital angle sensor with an uncertainty of $\pm 0.1^{\circ}$.

It is important to point out that the rotation of the tube could promote mixing of the fluid inside it, causing a perturbation on the flow field previous to the bubble. Perturbations can also be observed when gas is injected at the bottom of a tube filled with stagnant liquid. However, the bubble had sufficient length to develop its flow until the point where the measurements were carried out and to recover the liquid stagnation condition. Thus, the influence of the tube rotation on the obtained experimental results can be neglected.

The high speed ultrasonic system used to measure the bubble parameters consisted of a generator/multiplexer board, transducers, and a personal computer with a LabVIEW software developed

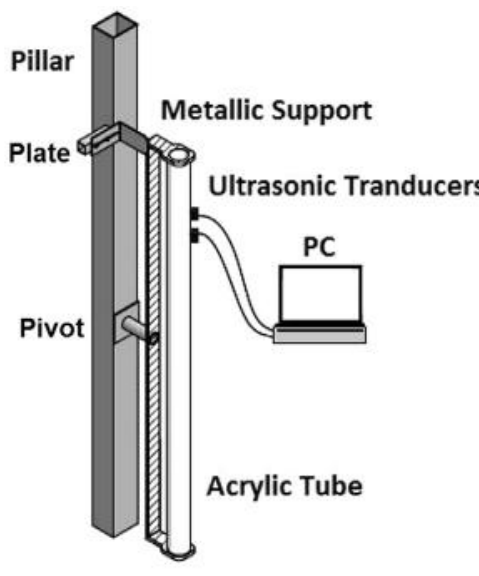

a)

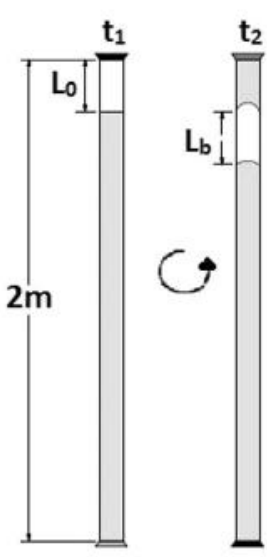

b)
Fig. 2. Schematic of the stagnant liquid vertical column: (a) Apparatus; (b) Bubble formation.

to control up to four transducers in pulse-echo or transmission mode. Two piezoelectric-type ultrasonic transducers of $10 \mathrm{MHz}$ and $6.35 \mathrm{~mm}$ diameter (Olympus Model V112) were mounted at 0.75 and $0.80 \mathrm{~m}$, respectively, at one side of the tube. The transducers were clamped on the outer surface of the tubes by means of supports, especially designed in order to assure the transducers alignment. Each support was fabricated according the tube dimensions. Details about the transducer supports can be viewed in De Azevedo (2016).

The LabView software controled an Ultratek board, model DSPUT5000-4, which was responsible for the signal generation, multiplexing, and data acquisition. The ultrasonic signals were digitized by the board, from each transducer, at a sampling rate of $100 \mathrm{MHz}$, corresponding to a time intervals of $10 \mathrm{~ns}$. The signal acquisition by the transducers was done with a time lag of $200 \mu \mathrm{s}$ for each channel of the board. The transducers signals, emitted and reflected back by the gas-liquid interface, were recorded by the computer in the form of spreadsheets files. The system was set up to record only ultrasonic signals with transit time lower than the time that the ultrasonic signal travelled along the inner tube diameter, when the tube was full with liquid. This procedure aimed to remove spurious signals, including multiple reflections arising from the opposite wall of the pipe.

The acquisition frequency during the experiments was $440 \mathrm{~Hz}$ with 2000 acquisition points, which resulted in an acquisition time of $4.5 \mathrm{~s}$. This time interval was sufficient for a complete detection of the bubbles, except for longer and/or slower bubbles rising in glycerin. In these cases, the acquisition frequency was reduced to $230 \mathrm{~Hz}$ in order to increase the acquisition time to $8.7 \mathrm{~s}$.

The pulse-echo ultrasonic technique is based on the high difference between the acoustic impedance of the gas and liquid phases, which allows that almost $99 \%$ of the emitted wave be reflected by a gas-liquid interface. Thus, the location of a gas-liquid interface can be determined by measuring the transit time between the emission of a wave and its return after being reflected (Chang and Morala, 1990).

To understand the signal processing involved, consider the Taylor bubble moving inside a tube, as shown in Fig. 3a and the corresponding ultrasonic waveform obtained in Fig. 3b. An initial pulse was periodically emitted by the ultrasonic transducer at a rate longer than the echo pulse transit times, $T_{1}, T_{2}, T_{3}, \ldots, T_{n}$, and fast enough to detect the moving gas-liquid interface. The individual values of $T$ were plotted against incident number $I_{n}$, determining the bubble shape, as presented in Fig. 3c. 


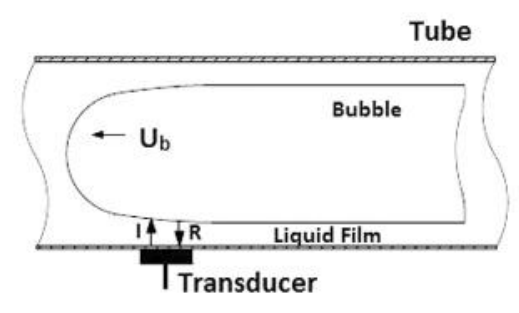

a)

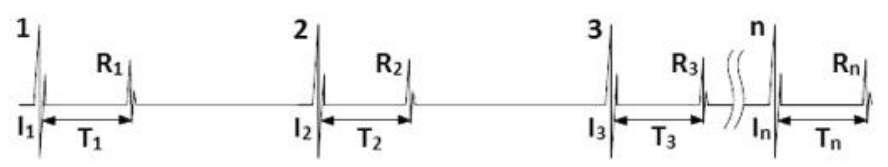

b)

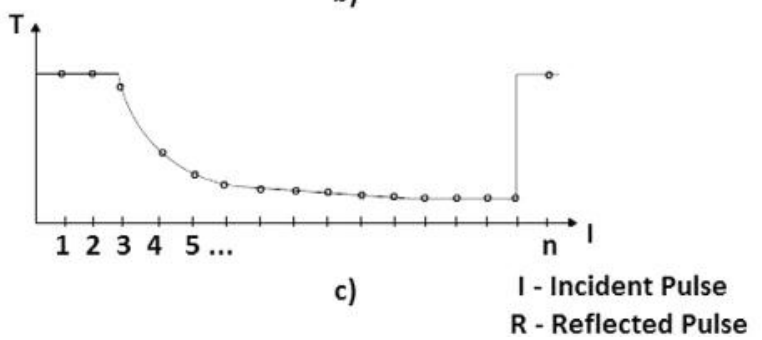

Fig. 3. Ultrasonic waveforms obtained in a typical gas-liquid interface of a Taylor bubble.

Thus, it was possible to measure the thickness $\delta$ of the falling film around a Taylor bubble by the relation:

$\delta=\frac{T_{n} c_{L}}{2}$,

where $T_{n}$ is the transit time corresponding to an incident number $I_{n}$ and $c_{L}$ is the sound velocity in the liquid phase.

In order of determining $\delta$, the sound velocity $c_{L}$ through the working liquids must be known. $c_{L}$ was determined by the relation:

$c_{L}=\frac{2 D}{T_{r 12}}$,

where $D$ is the tube inner diameter and $T_{r 12}$ is the measured transit time between the reflections that correspond to diametrically opposed inner walls of the tube fully filled with the liquid.

The bubble rising velocity was determined by the following relation:

$U_{b}=\frac{1}{N} \sum_{i=1}^{N} \frac{\Delta Z}{\tau_{i}}$,

where $N$ is the number of single bubbles studied, $\Delta Z$ is the distance between two transducers and $\tau_{i}$ is the time lag between the signals relating to the bubble detection by the two transducers, as presented in Fig. 4.

$\tau$ was obtained by cross correlation according to the following equation:

$R(\tau)=\int_{1}^{n} S_{1}\left(t_{n}\right) S_{2}\left(t_{n}+\tau\right) d t$

where $R$ is a function obtained by the cross correlation, $S_{1}(t)$ is the signal generated by the first transducer and $S_{2}(t)$ is the signal generated by the second transducer. The time lag was the time at which $\mathrm{R}$ takes a maximum value (Azaria and Hertz, 1984). The relative uncertainty of $U_{b}$ was estimated in $2 \%$, which lead to a maximum uncertainty of $2.3 \%$ for the computed $F r$.

In order of determining the development length $Z^{*}$ and the equilibrium film thickness $\delta_{e q}$, a MATLAB program was developed.
Initially, the nose and the tail of the Taylor bubble were identified from the transducer signals. Assigning the value of $n=1$ to the bubble nose, the program calculates the average value of the film thicknesses $m$ between two successive points:

$m=\frac{\delta_{n}+\delta_{n+i}}{2}$.

where $\delta_{n}$ is the film thickness at the position $n$ and $\delta_{n+i}$ is the film thickness at the position $n+i$. The analysis starts with $i=1$.

Then, the program calculates the relative discrepancy $d$ between the first point (bubble nose) and the average of the thicknesses calculated by Eq. (18):

$d=\frac{\left|\delta_{n}-m\right|}{m}$.

While the discrepancy $d$ is larger than a defined value, the program continues to increment $n$, repeating the process described by Eqs. (18) and (19), maintaining $i=1$.

When $d$ reaches a value less or equal to that pre-defined value, the program identifies the corresponding point $n$ and computed $m$, incrementing $\mathrm{i}(\mathrm{i}=2,3,4, \ldots)$. If the discrepancy $d$ remains less than or equal to the defined value until reaching the bubble tail, then that point $n$ is regarded as the acquisition point of the film development length $Z^{*}$ and the equilibrium film thickness $\delta_{e q}$ is calculated:

$\delta_{e q}=\frac{1}{n_{p}} \sum_{n_{Z^{*}}}^{n_{\text {tail }}} \delta_{n}$,

where $n_{Z^{*}}$ and $n_{\text {tail }}$ are the acquisition numbers corresponding to $Z^{*}$ and to the bubble tail, respectively. $n_{p}$ is the total number of acquisition points between $n_{Z^{*}}$ and $n_{\text {tail }}$.

In order to estimate the uncertainty of $\delta_{e q}$, we took into account the uncertainties concerning the calculations of $\delta$ by using Eq. (14), the dispersion of the $\delta_{e q}$ measured values for $N$ different bubbles defined by the standard deviations $\sigma_{\delta_{e q}}$, the small waves observed in the gas-liquid interfaces, defined by the average of the standard deviations of the measured values between $n_{Z^{*}}$ and $n_{\text {tail }}$ and the calibration curve presented in Fig. 5. All of these contributions lead to a $\delta_{e q}$ uncertainty estimated between $100 \mu \mathrm{m}$ and $280 \mu \mathrm{m}$, depending on the experimental conditions.

The calibration curve shown in Fig. 5 was obtained by using a cylindrical acrylic box, whose base was positioned at an horizontal surface, a pipette with a scale of $0.1 \pm 0.05 \mathrm{ml}$ and mixtures of water and glycerin. For each mixture used, the sound velocity $c_{L}$ in the liquid was determined by using Eq. (15), replacing the tube by a cylindrical box. A controlled volume of liquid was inserted into the box in order to obtain an uniform liquid film at its bottom. The expected film thickness was obtained dividing the inserted liquid volume by the base area of the acrylic box and the film thickness was obtained by using the pulse-echo ultrasonic technique.

The film development length $Z^{*}$ was determined by the relation:

$Z^{*}=U_{b}\left(n_{Z^{*}}-n_{\text {nose }}\right) \frac{t_{a c q}}{n_{a c q}}$,

where $n_{\text {nose }}$ is the acquisition number corresponding to the bubble nose, $t_{a c q}$ is the total acquisition time and $n_{a c q}$ is the total number of acquisition points obtained during the experiment. Note that $t_{a c q} / n_{\text {acq }}$ corresponds to the time lag between two consecutive points or incident numbers.

If the program identified, before reaching the tail, a discrepancy higher than that previously defined while $n$ was maintained at a fixed value and $i$ was incremented, then the search of the acquisition point of $Z^{*}$ has continued. $i$ returned to value 1 and $n$ was incremented until a new point with $d$ less than or equal to the defined value be found, restarting the procedure. 


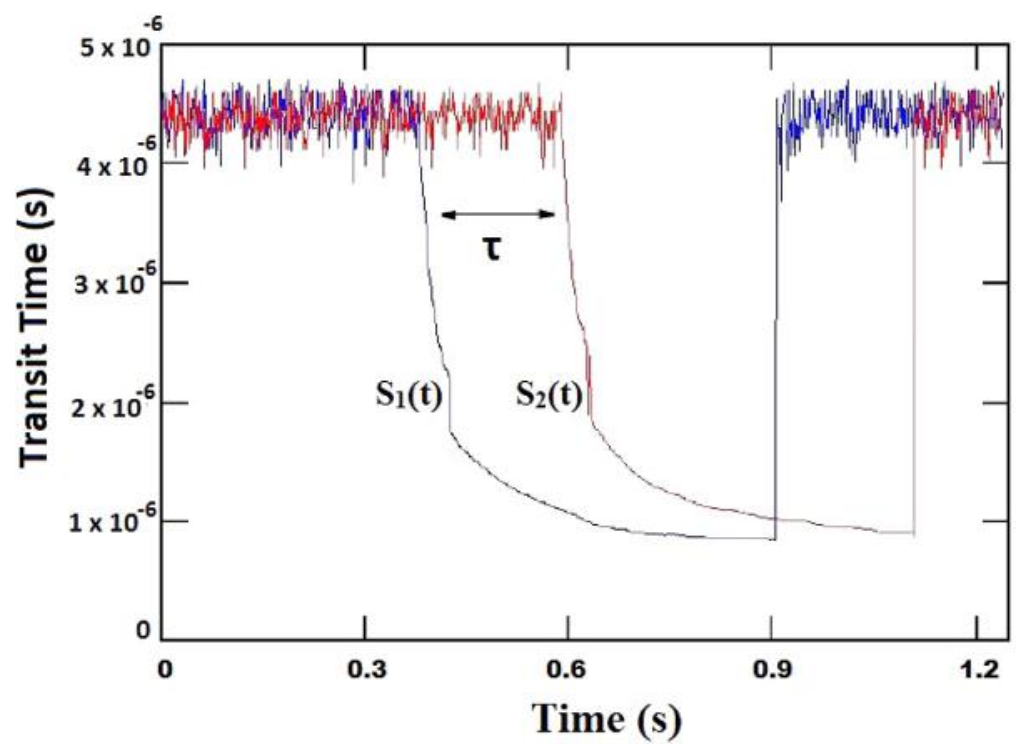

Fig. 4. Typical ultrasonic signals generated by two axially separated transducers.

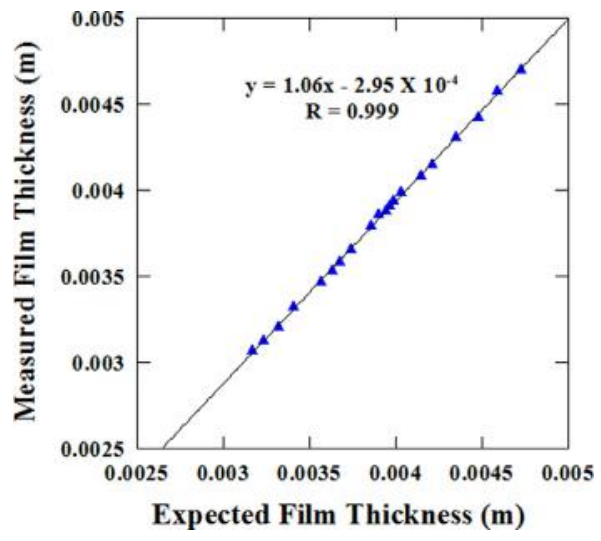

Fig. 5. Calibration curve of the ultrasonic system.

If the analysis has reached the bubble tail and any point has been identified, according to the criteria described above, it was considered that the full development of the liquid film was not reached.

For this work, the relative discrepancy was defined in the range $1 \% \leq d \leq 3 \%$, depending on the experimental conditions. It was observed that for higher viscosity liquids, or smaller $N_{f}$, the occurrence and intensity of waves at the gas-liquid interfaces were very small. On the other hand, when liquid viscosity was reduced or $N_{f}$ increased, the occurrence and intensity of the waves also increased. These waves can influence on the value and on the accuracy of $\delta_{\text {eq }}$ measurements by using Eq. (20) and consequently it can influence on the measurement of the film development length $Z^{*}$ by using the criteria described above. We verified that for almost all conditions studied, the program could identify the developed films defining $d$ between 1\% and 3\%. Only for the highest $N_{f}$ values, higher discrepancy values were required to satisfy the criteria previously described, which was not compatible with the idea of a constant film thickness. Under these conditions, we prefer to consider that the film did not reach its equilibrium condition.

Taking into account the uncertainties concerning the calculations of $Z^{*}$ by using Eq. (21) and the dispersion of measurements, characterized by the standard deviations computed for each experimental condition, the uncertainty of $Z^{*}$ was estimated in the range between $0.7 \mathrm{D}$ and $1.4 \mathrm{D}$.
Table 1

\begin{tabular}{llllll} 
Experimental conditions. \\
\hline$D(\mathrm{~m})$ & Liquid & $T\left({ }^{\circ} \mathrm{C}\right)$ & $\rho_{L}\left(\mathrm{~kg} / \mathrm{m}^{3}\right)$ & $\mu_{L}($ Pa.s $)$ & $N_{f}$ \\
\hline 0.019 & $100 \% \mathrm{~W}$ & 29.0 & 995.8 & 0.0008 & 10,000 \\
0.019 & $80 \% \mathrm{~W}+20 \% \mathrm{G}$ & 30.0 & 1058.3 & 0.0015 & 5,703 \\
0.019 & $50 \% \mathrm{~W}+50 \% \mathrm{G}$ & 30.0 & 1141.6 & 0.0057 & 1,638 \\
0.019 & $20 \% \mathrm{~W}+80 \% \mathrm{G}$ & 30.0 & 1214.1 & 0.0499 & 200 \\
0.019 & $100 \% \mathrm{G}$ & 30.0 & 1257.4 & 0.5979 & 17 \\
0.024 & $100 \% \mathrm{~W}$ & 24.0 & 997.1 & 0.0009 & 12,900 \\
0.024 & $80 \% \mathrm{~W}+20 \% \mathrm{G}$ & 26.0 & 1059.9 & 0.0017 & 7,321 \\
0.024 & $50 \% \mathrm{~W}+50 \% \mathrm{G}$ & 25.0 & 1144.2 & 0.0068 & 1,952 \\
0.024 & $20 \% \mathrm{~W}+80 \% \mathrm{G}$ & 26.0 & 1216.5 & 0.0629 & 224 \\
0.024 & $100 \% \mathrm{G}$ & 24.0 & 1261.3 & 0.9875 & 15 \\
0.034 & $100 \% \mathrm{~W}$ & 26.0 & 996.6 & 0.0009 & 22,422 \\
0.034 & $80 \% \mathrm{~W}+20 \% \mathrm{G}$ & 29.0 & 1058.7 & 0.0016 & 13,320 \\
0.034 & $50 \% \mathrm{~W}+50 \% \mathrm{G}$ & 28.0 & 1142.7 & 0.0061 & 3,650 \\
0.034 & $20 \% \mathrm{~W}+80 \% \mathrm{G}$ & 28.0 & 1215.3 & 0.0559 & 427 \\
0.034 & $100 \% \mathrm{G}$ & 28.5 & 1258.7 & 0.7035 & 35 \\
\hline
\end{tabular}

Percentages by volume (W-water; G-glycerin); $g=9.81 \mathrm{~m} / \mathrm{s}^{2}$

We studied a number of combinations of tube inner diameter $(D=0.019,0.024$ and $0.034 \mathrm{~m})$, working fluids (pure distilled water, pure glycerin and mixtures of them) and air pocket lengths $L_{0}$ (typically covering the range $0.01<L_{0}<0.60 \mathrm{~m}$, depending on the liquid used). For each of these conditions $\left(D\right.$, liquid and $\left.L_{0}\right)$, at least ten single bubbles were generated. Thus, the results presented correspond to average values of, at least, ten measurements of the desired parameters for each of these sets of data.

The fluid properties were calculated by using correlations given by Cheng (2008) that provide the density $\left(\rho_{L}\right)$ and the viscosity $\left(\mu_{L}\right)$ of the glycerin-water mixtures in the range of $0-100 \%$ and temperatures varying from 0 to $100^{\circ} \mathrm{C}$. The calculated $\mu_{L}$ was compared with three data bases (Chenlo et al., 2004; Segur and Oberstar, 1951; Shankar and Kumar, 1994) and the maximum average error of the predictions was $2.3 \%$. For $\rho_{L}$, the computed values were compared with a data base originally compiled by Miner and Dalton (1953), which led to errors smaller than $1 \%$.

The experimental conditions are summarized in Table 1.

\section{Results and discussions}

Fig. 6 presents the experimentally determined $\mathrm{Fr}$, for different $N_{f}$, together with the Nicklin et al. (1962) prediction for Fr of Tay- 


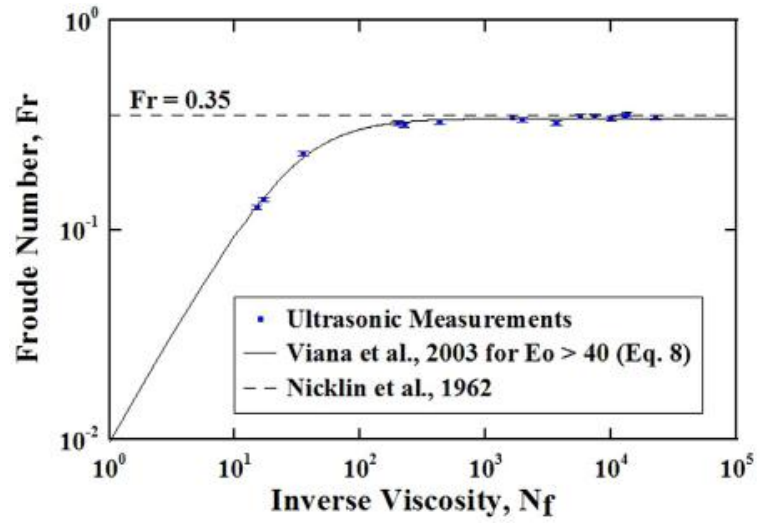

Fig. 6. Experimentally determined Fr, for different $N_{f}$, and the empirical correlation proposed by Viana et al. (2003), simplified for Eo > 40 (Eq. (8)).

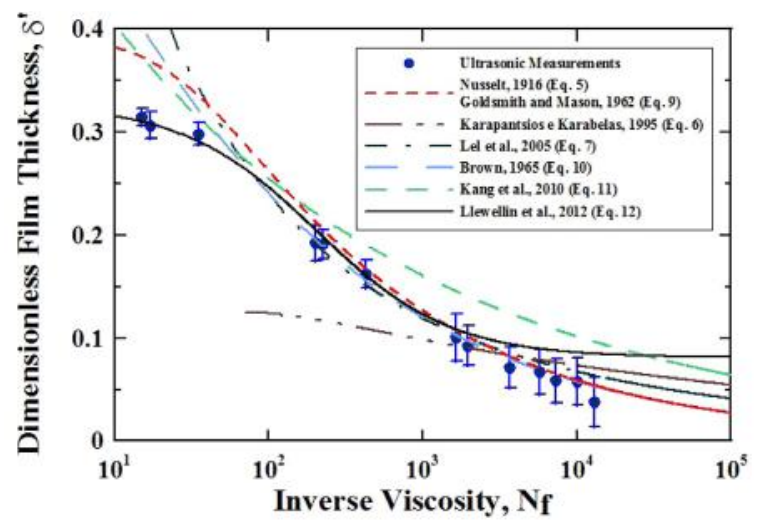

Fig. 7. Relationship $\delta_{e q}^{\prime}$ vs. $N_{f}$ and the correlations defined by Eqs. (5)-(7) and Eqs. (9)-(12).

lor bubbles rising in inviscid liquids and the empirical correlation defined by Eq. (8). It shows a very good agreement between the experimental measurements and the correlation of Viana et al. (2003), simplified for Eo > 40.

It seems that the pressure variation from the bottom to the top of the tubes does not exert a high influence on the movement of bubbles rising in stagnant liquids inside closed vertical tubes. Nicklin et al. (1962) showed that elongated bubbles of finite length rise relative to the liquid ahead of them at a velocity exactly equal to that presented previously by Dumitrescu (1943). When the tubes were sealed at the ends, no flow of liquid across a section ahead of the bubble was observed and bubbles of all lengths rise at a velocity defined by $U_{0}=0.35 \sqrt{g D}$. Only in a tube open at the top, the expansion of the bubble due to the change of static head as it rises gives the liquid above it an upward velocity. Since the bubble rises at the characteristic velocity $U_{0}$ relative to the liquid above it, the velocity in space is greater by an amount which depends on the length of the bubble. Fig. 6 also shows a very good agreement between the values measured of $\mathrm{Fr}$ and those predicted by Nicklin et al. (1962), for high $N_{f}$ conditions and closed tubes, which indicates a fully developed movement of the Taylor bubble and suggests that the pressure variation from the bottom to the top of the tube would not exert an influence on the results obtained in the present work.

Fig. 7 presents the dimensionless equilibrium thickness, $\delta_{e q}^{\prime}=\delta_{e q} / R$, measured by using the pulse-echo ultrasonic technique for different $N_{f}$ and the correlations defined by Eqs. (5)-(7) and Eqs. (9)-(12).

It can be seen that the Llewellin et al. (2012) correlation, Eq. (12), seems to overestimate the film thicknesses for higher

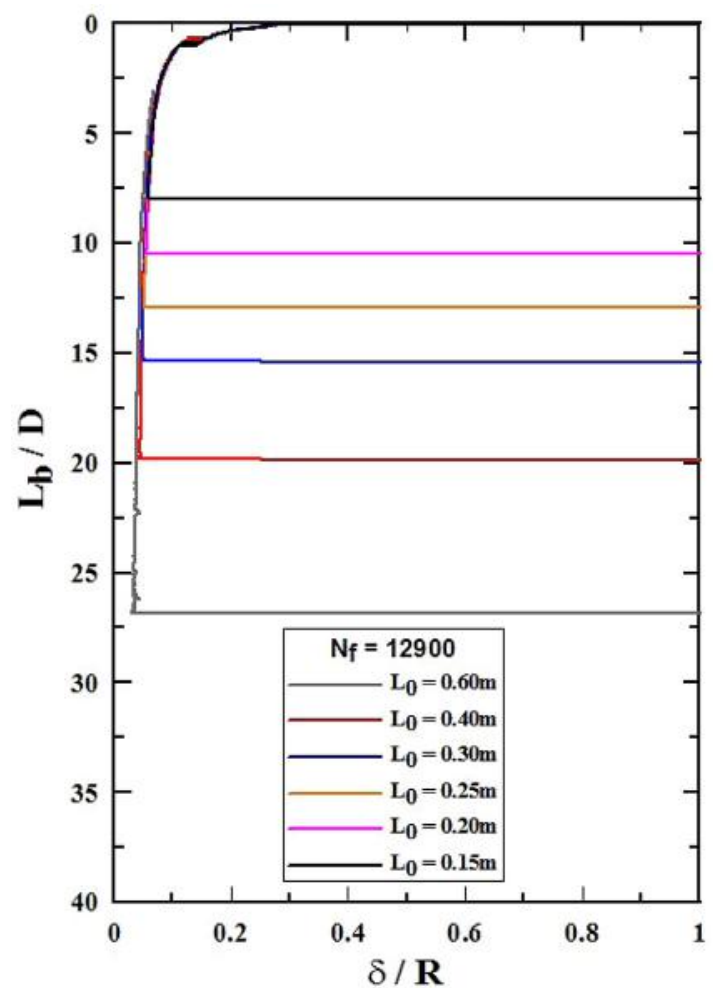

Fig. 8. Superposition of the profiles of Taylor bubbles rising in pure water inside a vertical tube of $\mathrm{D}=0.024 \mathrm{~m}\left(N_{f}=12900\right)$ generated from different air pocket $L_{0}$.

$N_{f}$. This fact can be attributed to an insufficient bubble length to allow the fully development of the liquid film falling around the bubble, as observed by De Azevedo et al. (2015) and De Azevedo (2016). When the bubble is not sufficiently long to allow that the film reaches the equilibrium condition, the methodology used by Llewellin et al. (2012), based on the relationship $L_{b}$ vs. $L_{0}$, tends to overestimate $\delta_{e q}$ and, probably, this is the case of the experiments performed by these authors in high $N_{f}$ conditions, since the maximum air pocket length $L_{0}$ used by them was $0.30 \mathrm{~m}$.

Fig. 8 shows that, for bubbles rising in distilled water inside a tube with $D=0.024 \mathrm{~m}$, the liquid film only reached the equilibrium for bubbles with $L_{b}>20 D$ (or for bubbles generated from $L_{0}$ $>0.40 \mathrm{~m}$ ), i.e., up to $L_{b} \approx 20 D$, the film remains thinning.

It can also be observed, in Fig. 8, a perfect superposition of the bubble profiles (gas-liquid interfaces) for Taylor bubbles generated from different $L_{0}$. This behavior was observed for all conditions studied, which indicates that an increment of the volume of Taylor bubbles rising in the same experimental conditions (liquid and internal tube diameter) did not change the bubble shape in the nose and film regions (Fig. 1). The bubble growth only occurs from its tail.

It is important to comment that the perfect superposition of the gas-liquid interfaces presented in Fig. 8, together with the very good agreement between the measured bubble velocities and the correlations presented to estimate them (Fig. 6), suggests that the tube rotation performed to generate the single bubble did not influence on the experimental results. Another evidence for this is the fact that the measured bubble velocities was basically the same for bubbles with different lengths $L_{b}$ rising in the same experimental conditions, which is in agreement with the behavior observed by Nicklin et al. (1962) for Taylor bubbles rising in closed vertical tubes.

Another important result obtained in the present work was that non fully developed films for $N_{f}>12900$ was observed, even 
Table 2

Summary of the correlations and their validity ranges.

\begin{tabular}{llll}
\hline Correlation & Original validity & Llewellin et al. (2012) & Present work \\
\hline Nusselt (1916) & $N_{f}<3,000$ & $N_{f}<3,000$ & $224<N_{f}<12,900$ \\
Lel et al. (2005) & $40<N_{f}<9,000$ & $40<N_{f}<9,000$ & $35<N_{f}<10,000$ \\
Karapantsios and Karabelas (1995) & $9,000<N_{f}<44,000$ & $2000<N_{f}<20,000$ & $1638<N_{f}<10,000$ \\
Kang et al. (2010) & $10<N_{f}<450$ & $N_{f} \approx 100$ & Poor agreement \\
Goldsmith and Mason (1962) & $N_{f}<3,000$ & $N_{f}<3,000$ & $224<N_{f}<12,900$ \\
Brown (1965) & $N_{f}>120$ & $50<N_{f}<3,000$ & $35<N_{f}<12,900$ \\
Llewellin et al. (2012) & - & $0.1<N_{f}<100,000$ & $15<N_{f}<5,703$ \\
\hline
\end{tabular}

for the highest bubble length studied in the present work $\left(L_{b}\right.$ $\approx 0.65 \mathrm{~m}$ ). Additionally, the measurements for $N_{f}=13320$ and $N_{f}=22422$ revealed strong ripples at the gas-liquid interface. These results were consistent with those of Mao and Dukler (1991) that reported a wavy structure at the free surface of liquid films falling around Taylor bubbles for $N_{f} \approx 35861$. Their experiments revealed that the film continued to accelerate for long distances behind the nose $\left(L_{b} \approx 11 D\right)$.

Fig. 7 also reveals a good agreement between the experimental values and those estimated by the Nusselt (1916) correlation, Eq. (5), with higher relative differences for lower $N_{f}$. This fact can be attributed to the thin film assumption, since a $N_{f}$ decrease is related with a combination of viscosity increase and tube diameter decrease. The best agreement range observed for the Nusselt (1916) correlation is $224<N_{f}<12900$. For the Karapantsios and Karabelas (1995) correlation, Eq. (6), it can be observed a good agreement between the correlation values and the measured ones, in the range $1638<N_{f}<10000$ and for the Lel et al. (2005) correlation, Eq. (7), a good agreement range was observed for $35<N_{f}<$ 10000. The Kang et al. (2010) correlation, Eq. (11), presented a very poor agreement with the experimental measurements achieved in the present work, which was also verified by Llewellin et al. (2012).

In Fig. 7, it can also be observed that the Goldsmith and Mason (1962), Eq. (9), and Brown (1965), Eq. (10), correlations agree well with the experimental data for higher $N_{f}$, but the relative errors between the experimental and the estimated values tend to increase when $N_{f}$ decrease. In the Goldsmith and Mason (1962) correlation, this behavior can be attributed to the thin-film assumption used in its theoretical development. On the other hand, the Brown (1965) correlation doesn't make use of thin-film assumption, but it was assumed a constant ratio $U_{b} / \sqrt{g\left(D-2 \delta_{e q}\right)}$, which was not verified for the lowest values of $N_{f}$ and can explain the observed disagreement.

Table 2 summarizes the $N_{f}$ ranges for which the correlations evaluated in this work agreed well with the experimental measurements, along with the original validity ranges defined by their authors and rewritten as a function of $N_{f}$. It can be observed that all correlations presented in Table 2 can be used to estimate the thickness of liquid films falling around Taylor bubbles, except the Kang et al. (2010) correlation. However, all of them showed limited $N_{f}$ ranges for their applications.

Based on the experimental results obtained in the present work, we proposed an new empirical correlation to estimate the dimensionless equilibrium film thickness $\delta_{e q}^{\prime}$ for $N_{f}$ ranging from 15 to 12900 . This correlation was obtained from the best fit of the experimental measurements and given by:

$\delta_{\text {eq }}^{\prime}=-4.19 \times 10^{-2} \ln N_{f}+4.25 \times 10^{-1}$.

It is not recommended to extend the validity of the correlation for $N_{f}>12900$, because no evidence was found about fully developed films around Taylor bubbles rising in those conditions. At the lower limit, an extension was limited by the experimental conditions used in this work. Fig. 9 shows that the correlation given by Eq. (22) agrees well with the experimental data, including those obtained by Llewellin et al. (2012) and Nogueira et al.

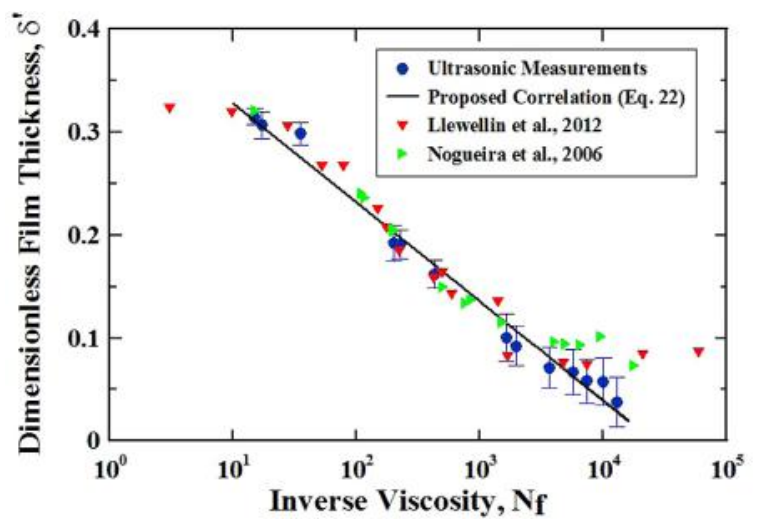

Fig. 9. Dimensionless equilibrium thickness $\delta_{e q}^{\prime}$ of the falling film of liquid around a Taylor bubble as a function of the inverse viscosity number $N_{f}$.

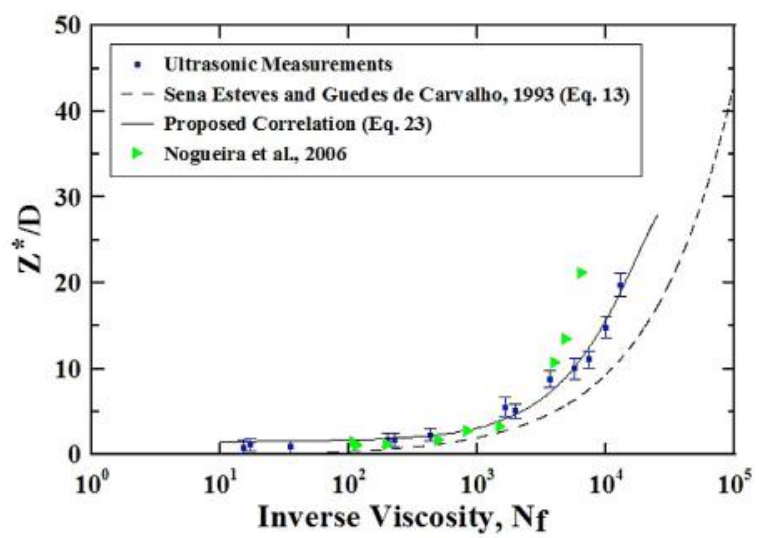

Fig. 10. The dimensionless film development length $Z^{*} / D$ a function of the inverse viscosity number $N_{f}$.

(2006), except for high values of $N_{f}$ and especially outside the validity range of the proposed correlation.

The measured values of $Z^{*} / D$ by using the ultrasonic technique and those estimated by the Sena Esteves and Guedes de Carvalho (1993) correlation, Eq. (13), are presented in Fig. 10. The experimental data confirmed the qualitative behavior expected by the Sena Esteves and Guedes de Carvalho (1993) correlation, with a strong dependence of $Z^{*} / D$ with $N_{f}$, indicating that longer bubble lengths are required to allow that the films reach their equilibrium condition, when $N_{f}$ increases.

Based on the experimental results of the present study, an empirical correlation to estimate $Z^{*}$ was also proposed for $N_{f}$ ranging from 15 to 12900 :

$\frac{Z^{*}}{D}=1.51+1.70 \times 10^{-3} N_{f}-2.45 \times 10^{-8} N_{f}^{2}$.

The good agreement of Eq. (23) with the experimental data can also be observed in Fig. 10, where the measurements performed 
in the present work can be observed together with those performed by Nogueira et al. (2006). The experimental values of $Z^{*} / D$ measured by Nogueira et al. (2006) were obtained from a graph $\left[\left(Z^{*} / D\right)_{\exp } /\left(Z^{*} / D\right)_{\text {theo }}\right]$ vs. $\operatorname{Re}_{f}$ and from data provided in the work, where $\left(Z^{*} / D\right)_{\text {exp }}$ are the experimental measurements and $\left(Z^{*} / D\right)_{\text {theo }}$ are the theoretical values estimated by the following correlation proposed by Campos and Guedes de Carvalho (1988):

$\frac{Z^{*}}{D}=\frac{\left[\left(g \delta_{e q}^{2} / 2 v_{L}\right)+U_{b}\right]^{2}}{2 g D}$,

where $v_{L}$ is the liquid kinematic viscosity. It is important to note that Eq. (24) is equivalent to Eq. (13), but written in a dimensional form.

The strong dependence of $Z^{*} / D$ in $N_{f}$ observed in Fig. 10 can explain the underdeveloped films observed for $N_{f}=13320$ and $N_{f}=22422$. It can be verified that for these cases, very long bubble lengths were required to allow the fully development of the films, which exceeded the maximum $L_{b}$ studied in the present work. However, because of the strong ripples observed at the gas-liquid interfaces in those conditions, it is not possible to assure that the films will really reach their equilibrium.

Earlier, other authors had studied the films around Taylor bubbles by measuring their velocity profiles and the bubble shapes. However, the bubble length studied was insufficient to allow that the films reach their equilibrium. Van Hout et al. (2002) and Polonsky et al. (1999) studied bubbles with length $L_{b}$ up to $3.6 D$ and $6.4 D$, respectively, with $N_{f} \approx 12500$, and verified that the films remained underdeveloped under these conditions. Ahmad et al. (1998) studied Taylor bubbles with $L_{b} \approx 4.5 D$ rising in kerosene, inside a vertical tube with $0.025 \mathrm{~m}$ inner diameter $\left(N_{f} \approx 4200\right)$ and observed that the measured velocity profile was characteristic of a developing film.

Nogueira et al. (2006) performed experiments for $N_{f}$ ranging from 15 to 18000 , but the air volumes, reported by them, were not sufficient to generate bubbles with lengths that allow the fully development of the film for the highest $N_{f}$.

Llewellin et al. (2012) experiments were conducted with longer bubbles for $N_{f}$ ranging from 0.2 to 59000 , however, the graphical methodology applied by them resulted in an overestimation of $\delta_{\text {eq }}$, especially for higher values of $N_{f}$, as commented earlier. Furthermore, no evidence was presented that the films really reach the equilibrium for higher values of $N_{f}$.

Thus, the experiments performed in the present work, with bubble lengths $L_{b}$ sufficiently long to allow the fully development of the films, in a wide range of $N_{f}$, can lead to a better understanding of the behavior of liquid films around Taylor bubbles. Since this behavior is directly related with mass and heat transfer processes and with the mechanisms of bubble interactions in slug flow, the results reported are relevant also for the validation of CFD codes for simulation of this flow pattern (Kang et al., 2010; Lu and Prosperetti, 2009; Ramdin and Henkes, 2012).

\section{Conclusions}

The non-intrusive pulse-echo ultrasonic technique was employed to measure different parameters of the falling liquid film around single Taylor bubbles rising in vertical stagnant liquid columns for inverse viscosity number $N_{f}=\rho_{L} \sqrt{g D^{3}} / \mu_{L}$ ranging from 15 to 22422 . The measured bubble velocities $U_{b}$ showed good agreement with the correlation of Viana et al. (2003), simplified for $E o>40$ (Eq. (8)).

The measurements of the bubble shape, $\delta_{e q}$ and $Z^{*}$ achieved in the present work indicated that the liquid films falling around Taylor bubbles rising in stagnant liquids inside vertical tubes tend to reach an equilibrium condition where their thicknesses $\delta$ can be considered as constant (fully developed films). However, the bubble lengths $L_{b}$ required for this revealed to be strongly dependent on $N_{f}$. Increasing $N_{f}$, much longer bubbles are required to allow the fully development of the film, which is in agreement with the qualitative behavior predicted by Sena Esteves and Guedes de Carvalho (1993), Eq. (13). Fully developed films were not observed for $N_{f}>12900$.

Based on the experimental data obtained in the present work, some correlations used to estimate the thickness of the falling film of liquid $\delta$ around a Taylor bubble were evaluated and the application range of $N_{f}$ for each of them was suggested. Moreover, new empirical correlations were proposed to estimate the dimensionless equilibrium film thickness $\delta_{e q}^{\prime}$ and film development length $Z^{*} / D$ (Eqs. (22) and (23)) respectively.

\section{Acknowledgments}

The authors are grateful to CNPq, FINEP and FAPERJ for the financial support.

\section{References}

Ahmad, W.R., De Jesus, J.M., Kawaji, M., 1998. Falling film hydrodynamics in slug flow. Chem. Eng. Sci. 53 (1), 123-130.

Azaria, M., Hertz, D., 1984. Time delay estimation by generalized cross correlation methods. IEEE Trans. Acoust. Speech Signal Process. ASSP32 (2), 280-285.

Brown, R.A.S., 1965. Mechanics of large gas bubbles in tubes 0.1. bubble velocities in stagnant liquids. Can. J. Chem. Eng. 43 (5), 217-223.

Campos, L.B.L.M., Guedes de Carvalho, J.R.F., 1988. An experimental study of the wake of gas slugs rising in liquids. J. Fluid Mech. 196, 27-37.

Chang, J.S., Morala, E.C., 1990. Determination of two-phase interfacial areas by ultrasonic technique. Nuclear Eng. Des. 122, 143-156.

Cheng, N.S., 2008. Formula for the viscosity of a glycerol-water mixture. Ind. Eng. Chem. Res. 47 (9), 3285-3288

Chenlo, F., Moreira, R., Pereira, G., Bello, B., 2004. Kinematic viscosity and water activity of aqueous solutions of glycerol and sodium chloride. Eur. Food Res. Technol. 219 (4), 403-408.

Davies, R.M., Taylor, G., 1950. The mechanics of large bubbles rising through extended liquids and through liquids in tubes. Proc. Royal Soc. London Series A 200 (1062), 375-390

De Azevedo, M.B., 2016. Experimental Studies of Single Taylor Bubbles Rising in Vertical and Slightly Inclined Tubes. Programa de Engenharia Nuclear/COPPE/UFRJ, Rio de Janeiro, Brazil D.Sc. Thesis.

De Azevedo, M.B., Faccini, J.L.H., Su, J., 2015. Experimental study of single taylor bubbles rising in stagnant liquid mixtures inside of vertical tubes.. In: Proceedings of the 2015 International Nuclear Atlantic Conference - INAC2015. Sao Paulo, Brazil.

Dumitrescu, D.T., 1943. Flow on a air bubble in the vertical tube. Zeitschrift Fur Angewandte Mathematik Und Mechanik 23, 139-149.

Ghajar, A.J., 2005. Non-boiling heat transfer in gas-liquid flow in pipes - a tutorial J. Braz. Soc. Mech. Sci. Eng. 27 (1), 46-73.

Goldsmith, H.L., Mason, S.G., 1962. The movement of single large bubbles in closed vertical tubes. J. Fluid Mech. 14 (1), 42-58.

Kang, C.-W., Quan, S., Lou, J., 2010. Numerical study of a Taylor bubble rising in stagnant liquids. Phys. Rev. E 81 (6), 066308

Karapantsios, T.D., Karabelas, A.J., 1995. Longitudinal characteristics of wavy falling films. Int. J. Multiphase Flow 21, 119-127.

Lel, V.V., Al-Sibai, F., Leefken, A., Renz, U., 2005. Local thickness and wave velocity measurement of wavy films with a chromatic confocal imaging method and fluorescence intensity technique. Exp. Fluids 39 (5), 856-864.

Llewellin, E.W., Del Bello, E., Taddeucci, J., Scarlato, P., Lane, S.J., 2012. The thickness of the falling film of liquid around a taylor bubble. Proc. Royal Soc. A - Math Phys. Eng. Sci. 468, 1041-1064.

Lu, X.Z., Prosperetti, A., 2009. A numerical study of taylor bubbles. Ind. Eng. Chem. Res. 48 (1), 242-252.

Mao, Z.-S., Dukler, A., 1991. The motion of Taylor bubbles in vertical tubes-II. Experimental data and simulations for laminar and turbulent flow. Chem. Eng. Sci. 46 (8), 2055-2064.

Matikainen, L., Irons, E.C., Morala, E.C., Chang, J.S., 1985. An Itrasonic measuring system for submerged gas injection. In: AIME Proceedings 5th Process Technology Conference, vol. 5 , pp. 65-77.

Miner, C.S., Dalton, N.N., 1953. Glycerol (American Chemical Society Monography 117). Reinhold Publishing Corp., New York.

Murai, Y., Tasaka, Y., Nambu, Y., Takeda, Y., Gonzalez, S.R., 2010. Ultrasonic detection of moving interfaces in gas-liquid two-phase flow. Flow Meas. Instrum. 21 (3) 356-366.

Nicklin, D., Wilkes, J., Davidson, J., 1962. Two-phase flow in vertical tubes. Trans. Instn. Chem. Engr 40, 61-68. 
Nogueira, S., Riethmuller, M.L., Campos, J.B.L.M., 2006. Flow in the nose region and annular film around a taylor bubble rising through vertical columns of stagnant and flowing newtonian liquids. Chem. Eng. Sci. 61, 845-857.

Nusselt, W., 1916. Die oberflachenkondesantion des wasserdamphes. Zeitschrift des Vereines Deutschem Ingenieure 60, 541-546.

Park, H.J., Tasaka, Y., Murai, Y., 2015. Ultrasonic pulse echography for bubbles traveling in the proximity of a wall. Meas. Sci. Technol. 26 (12), 125301.

Polonsky, S., Shemer, L., Barnea, D., 1999. The relation between the Taylor bubble motion and the velocity field ahead of it. Int. J. Multiphase Flow 25, 957-975.

Rahim, R.A., Rahiman, M.H.F., Chan, K.S., Nawawi, S.W., 2007. Non-invasive imaging of liquid/gas flow using ultrasonic transmission-mode tomography. Sensors and Actuators A 135 (2), 337-345.

Ramdin, M., Henkes, R., 2012. Computational fluid dynamics modeling of benjamin and taylor bubbles in two-phase flow in pipes. J. Fluids Eng.-Trans. ASME 134 (4), 041303.
Segur, J.B., Oberstar, H.E., 1951. Viscosity of glycerol and its aqueous solutions. Ind. Eng. Chem. 43 (9), 2117-2120.

Sena Esteves, M.T., Guedes de Carvalho, J.R.F., 1993. Liquid-side mass transfer coefficient for gas slugs rising in liquids. Chem. Eng. Sci. 20, 3497-3506.

Shankar, P.N., Kumar, M., 1994. Experimental determination of the kinematic viscosity of glycerol-water mixtures. Proc. Royal Soc. A 444 (1922), 573-581.

Van Hout, R., Gulitski, A., Barnea, D., Shemer, L., 2002. Experimental investigation of the velocity field induced by a Taylor bubble rising in stagnant water. Int. J. Multiphase Flow 28, 579-596.

Viana, F., Pardo, R., Ynez, R., Trallero, J.L., Joseph, D.D., 2003. Universal correlation for the rise velocity of long gas bubbles in round pipes. J. Fluid Mech. 494, 379-398. Wallis, G.B., 1969. One-Dimensional Two-Phase Flow. McGraw-Hill, New York. 